WISSENSCHAFTLICH-TECHNISCHE BERICHTE FZR-256

April 1999

ISSN 1437-322X

\title{
Preprint
}

Wolfgang Matz*, Norbert Schell, Gert Bernhard, Jürgen Claußner, Winfried Oehme, Friedrich Prokert, Tobias Reich, Reiner Schlenk, Dieter Pröhl, Harald Funke, Frank Eichhorn, Manfred Betzl, Siegfried Dienel, Vincenz Brendler, Melissa A. Denecke, Hans Krug, Wolfgang Neumann, Gudrun Hüttig, Peter Reichel, Udo Strauch

\section{ROBL - a CRG Beamline for Radiochemistry and Materials Research at the ESRF}


Herausgeber:

FORSCHUNGSZENTRUM ROSSENDORF

Postfach 510119

D-01314 Dresden

Telefon +493512600

Telefax +493512690461

http://www.fz-rossendorf.de/

Als Manuskript gedruckt

Alle Rechte beim Herausgeber 


\section{FORSCHUNGSZENTRUM ROSSENDORF}

WISSENSCHAFTLICH-TECHNISCHE BERICHTE

FZR-256

April 1999

\section{Preprint}

Wolfgang Matz*, Norbert Schell, Gert Bernhard, Jürgen Claußner, Winfried Oehme, Friedrich Prokert, Tobias Reich, Reiner Schlenk, Dieter Pröhl, Harald Funke, Frank Eichhorn, Manfred Betzl, Siegfried Dienel, Vincenz Brendler, Melissa A. Denecke, Hans Krug, Wolfgang Neumann, Gudrun Hüttig, Peter Reichel, Udo Strauch

\section{ROBL - a CRG Beamline for Radiochemistry and Materials Research at the ESRF}




\begin{abstract}
The paper describes the Rossendorf Beamline (ROBL) built by the Forschungszentrum Rossendorf at the ESRF. ROBL comprises two different and independently operating experimental stations: a radiochemistry laboratory for $\mathrm{X}$-ray absorption spectroscopy of non-sealed radioactive samples and a general purpose materials research station for $X$-ray diffraction and reflectometry mainly of thin films and interfaces modified by ion beam techniques. The radiochemistry set-up is worldwide an unique installation at a modern synchrotron radiation source.
\end{abstract}

Keywords: $\quad$ synchrotron radiation beamline, EXAFS, XANES, radiochemistry laboratory, radionuclides, $\mathrm{X}$-ray diffraction and reflectometry, thin films, interfaces, melts

\title{
Zusammenfassung
}

Der Bericht beschreibt die Rossendorfer Beamline (ROBL), die vom Forschungszentrum Rossendorf an der ESRF errichtet wurde. ROBL besteht aus zwei unabhängigen Meßplätzen: einem kleinen radiochemischen Labor für Röntgen- Absorptionsspektroskopie an offenen radioaktiven Proben und einem Vielzweckmeßplatz für Materialuntersuchungen insbesondere mit Röntgendiffraktion und Reflektometrie. Der Radiochemie-Meßplatz ist eine weltweit unikale Einrichtung an einer modernen Synchrotronstrahlungsquelle.

* Corresponding author: tel. +49-351-260 3122; fax +49-351-260 3438;

E-mail:w.matz@fz-rossendorf.de 


\section{Introduction}

The ROssendorf BeamLine (ROBL) is a collaborating research group (CRG) beamline at the European Synchrotron Radiation Facility (ESRF). ROBL has been designed for performing experiments on two different experimental stations located in lead shielded hutches: a Radiochemistry Hutch (RCH) and a Materials Research Hutch (MRH). X-ray absorption spectroscopy and X-ray diffraction and reflectometry are the main experimental techniques used in $\mathrm{RCH}$ and $\mathrm{MRH}$, respectively. Both end-stations operate alternatively.

The beamline was built and is operated by the Forschungszentrum Rossendorf (FZR) located near Dresden. Most of the beamtime is used by the FZR for inhouse research devoted to:

- Radioecological research as scientific background for risk assessment and development of remediation strategies for areas contaminated by radionuclides. Determination of the chemical speciation of radionuclides interacting with geological material, natural and anthropogenic organics, and microorganisms. Study of the influence of these interactions on radionuclide migration and retardation in the environment.

- Structural identification and characterisation (including texture) of modifications of surfaces and interfaces produced by ion beam techniques for applications as hard covers, sensors or in semiconductor technology. Study of interfaces in thin films and nanometer-multilayers. Structural investigations of melts and amorphous solids.

The beamline is also available to outside users to perform experiments either in collaboration with the FZR or by submitting a proposal to the ESRF. One third of the ROBL beamtime will be allocated by the ESRF for peer-reviewed experiments.

This paper describes the beamline design, the technical specifications and the standard experimental equipment of both experimental stations.

\section{Beamline optics}

The beamline is located at the bending magnet port BM 20 of the ESRF. The overall layout of ROBL is shown in Fig. 1. The beamline optics uses horizontally a fan of $2.8 \mathrm{mrad}$ of synchrotron radiation from the hard edge of the ESRF bending magnet ( $0.8 \mathrm{~T}$ range, critical energy $19.6 \mathrm{keV})$ [1]. The layout of the optics with the distances from the source point is sketched in Fig. 2. The general concept is a double crystal monochromator of fixed-exit type located between two mirrors. The beamline is designed for an energy range from 5 to $35 \mathrm{keV}$. The lower energy limit is given essentially by the mandatory Bewindows. The upper energy limit was chosen to cover at least one absorption edge to allow XAS experiments on all chemical elements beginning from $\mathrm{Ti}$ onward. 

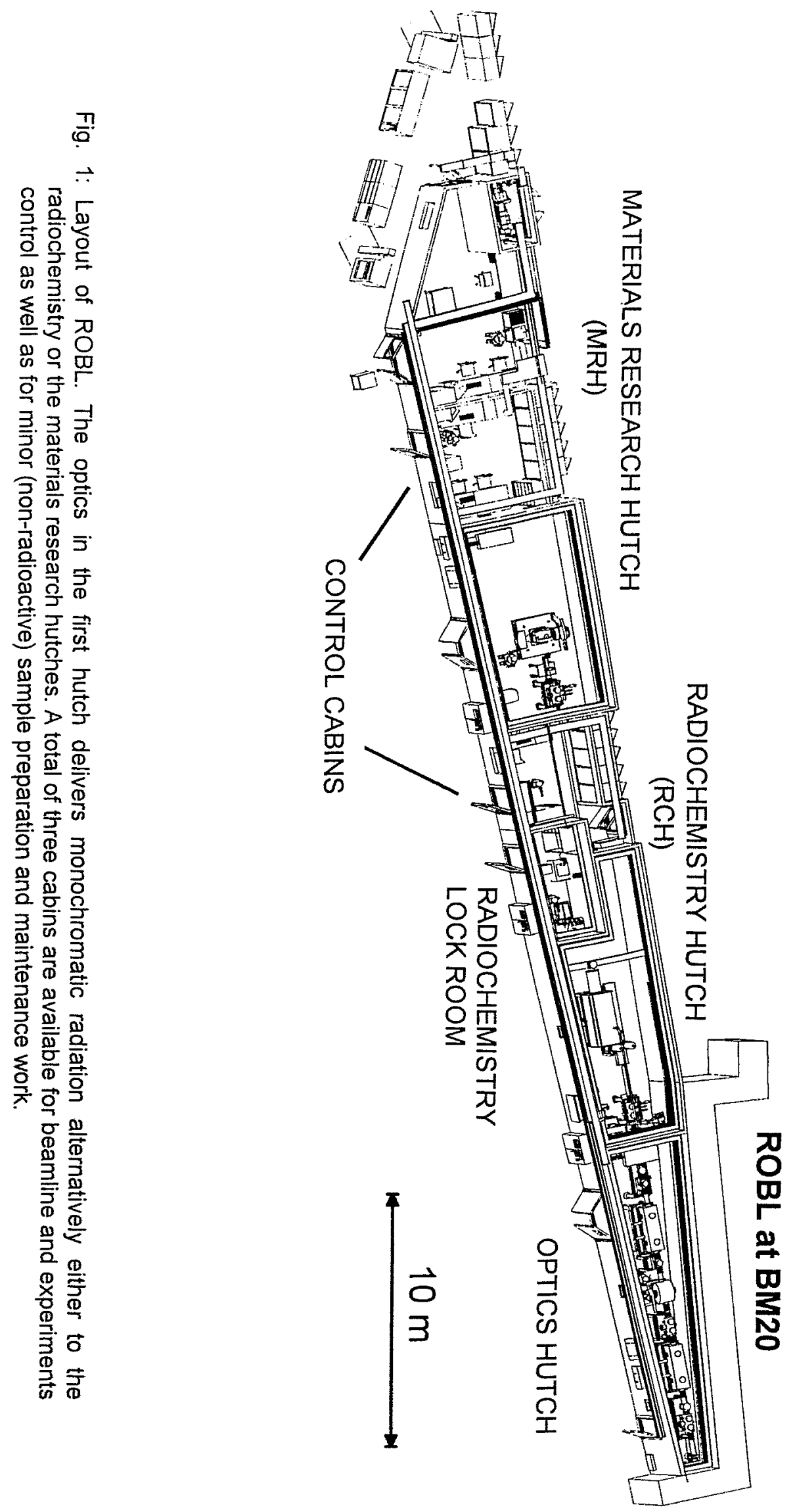

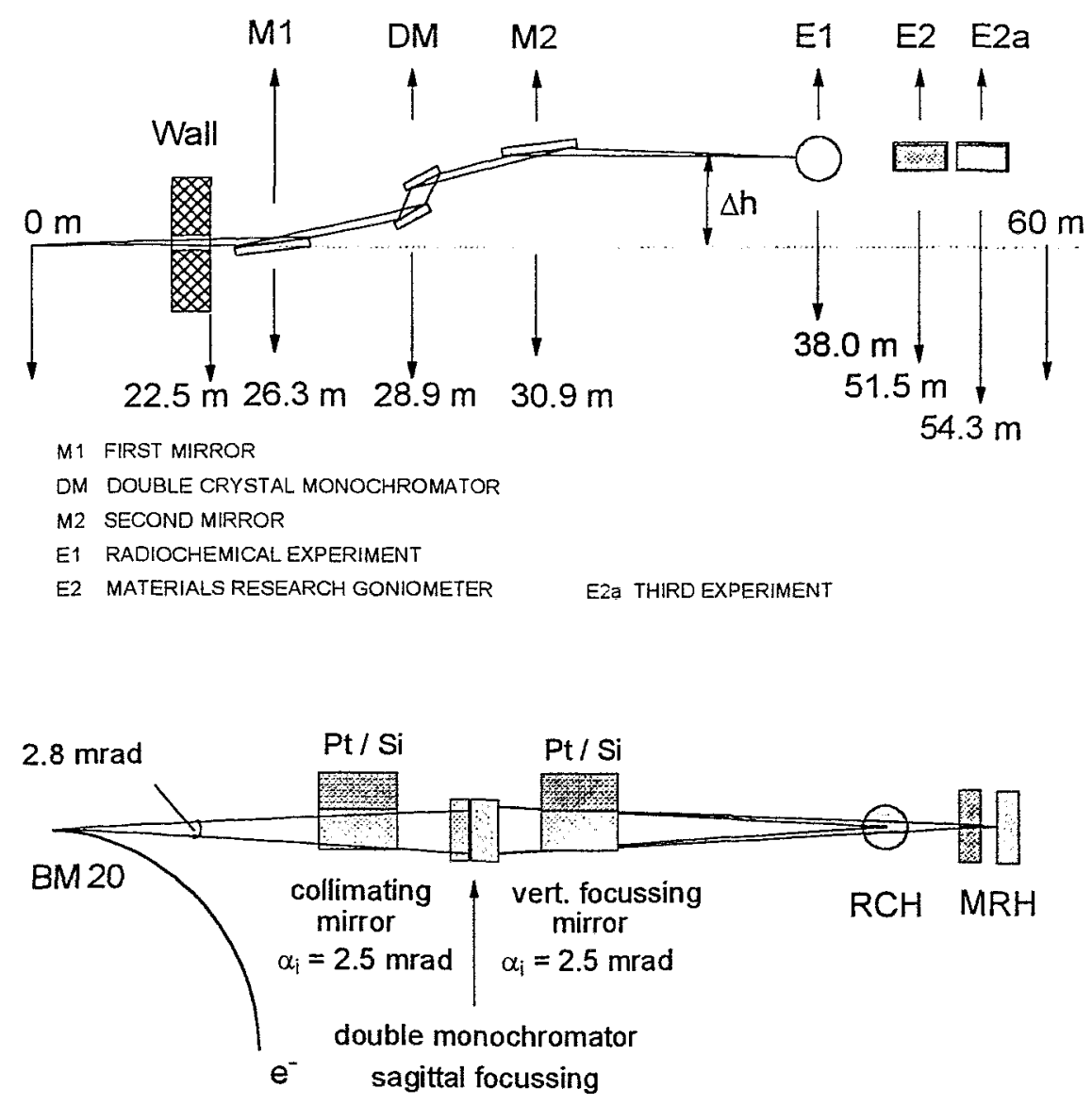

Fig. 2: Scheme of the X-ray optics of ROBL. A mirror - double crystal monochromator - mirror arrangement conditions the beam for the two experimental end-stations.

\subsection{X-ray mirrors}

The two mirror design with the identical fixed incident angle for both mirrors was adopted to suppress the higher-order harmonics in the monochromatic beam coming from high-energy radiation, to reduce the heat load on the monochromator, and to have a parallel or vertically focussed beam at the experimental stations always at the same height. The latter was dictated by safety requirements for the radiochemistry hutch (cf. 3.2). The most important energy regions for the main applications of ROBL are below $10 \mathrm{keV}$ and between 16 and $22 \mathrm{keV}$, respectively. To minimise disturbations from absorption edges in these energy regions by mirror coatings, the mirror substrates were coated with parallel stripes of silicon and platinum each.

With a fixed angle of incidence of $2.5 \mathrm{mrad}$ and a length of $1200 \mathrm{~mm}$, the first mirror accepts vertically at $12 \mathrm{keV}$ about $55 \%$ and at $25 \mathrm{keV}$ about $72 \%$ of the photons from the source. The maximum beam height limited by the mirror 
design is $3 \mathrm{~mm}$. The calculated spectral transmission for the two cases is shown in Fig. 3. In the energy region below $10 \mathrm{keV}$, the transmission is determined by the absorption of beryllium windows calculated from experimental values [2]. The harmonic suppression is better than $8 \times 10^{-4}$ for all energies accessible with the silicon stripes. For the platinum stripes, the harmonic suppression is of the same order of magnitude for all energies above $13.5 \mathrm{keV}$.

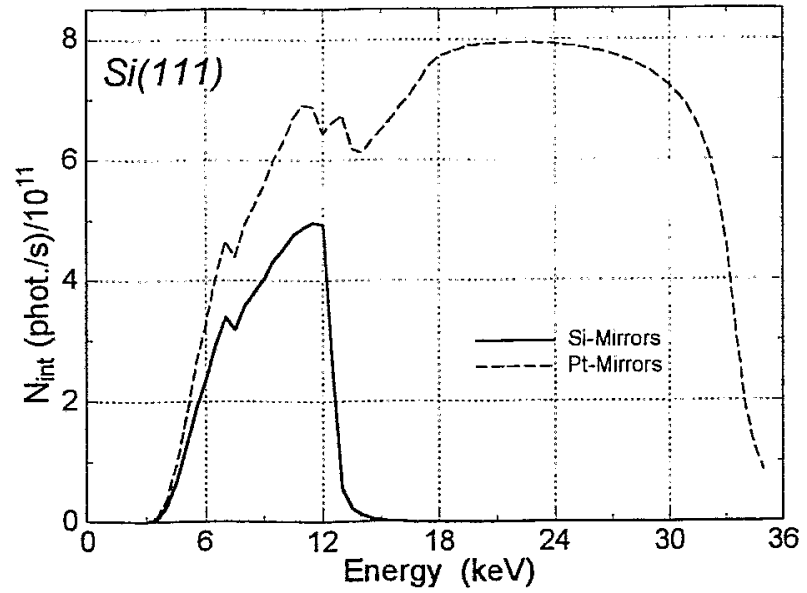

Fig. 3

Calculated flux of focussed radiation with silicon and platinum coated mirrors and silicon (111) crystals in the double crystal monochromator using the SHADOW code [4]. The attenuation of $1.5 \mathrm{~mm}$ beryllium from the windows is included. Geometrical reasons of the monochromator design limit the accesible energy range for $\mathrm{Si}(111)$ crystals to about $25 \mathrm{keV}$.

The mirrors including cooling and bending mechanisms were purchased completely (Zeiss) [3]. The first mirror is made of a single Si crystal which is water cooled from the side. The second mirror uses a ZERODUR substrate without cooling. Both mirrors are equipped with pneumatic benders. The first mirror is bent with a radius of $20.8 \mathrm{~km}$ to collimate the incident radiation onto the first monochromator crystal. The second mirror with an adjustable bending radius down to $8 \mathrm{~km}$ focusses the beam vertically to the two experimental endstations.

\subsection{Double crystal monochromator}

The double crystal monochromator of fixed-exit type with a beam offset of 18 $\mathrm{mm}$ operates with either $\mathrm{Si}(311)$ or $\mathrm{Si}(111)$ crystal sets. The design is mainly a commercial one (Oxford Instruments, ATG). The axis of a high-precision rotation table is fed into a vacuum vessel. A crystal cage mounted on this axis carries both crystals. The first crystal is mounted with the surface in the rotation axis while the second crystal can be moved parallel and perpendicular to the beam direction. The combined motion of both drives realises a fixed-exit beam independent of the selected energy. The mechanical construction allows to use the $\mathrm{Si}(111)$ crystal pair up to $25 \mathrm{keV}$ and the Si(311) crystal pair up to $35 \mathrm{keV}$. The first crystal is manually adjusted, while all movements of the second crystal are motorised and remote controlled. So the alignment with the beam and a fine tuning by piezoelectric translators is possible at any time. 
The first crystal is directly water cooled. The cooling water is supplied by individual jets directed into slots machined in the back of the crystal. Bridges between the cavities provide stability of the reflecting crystal surface. In order to reduce bulging by too high water pressure, to avoid drifts in water pressure, and to have good temperature stability the cooling consists of a two stage circuit system. The second crystal will be equipped with a bender (ESRF design) to permit sagittally focussing. In the beginning this will be available only for Si(311).

For Quick-EXAFS, with a fixing of the second crystal a pseudo channel-cut mode of the monochromator can be realised. For energies above $14 \mathrm{keV}$ the beam height variation during a $500 \mathrm{eV}$ wide scan in pseudo channel-cut mode is less than $0.6 \mathrm{~mm}$.

The calculated energy resolution for the described arrangement of the main optical elements is shown in Fig. 4. This resolution allows to study XANES at all edges within the accessible energy range. The measured energy resolution is within $20 \%$ of the calculated values.

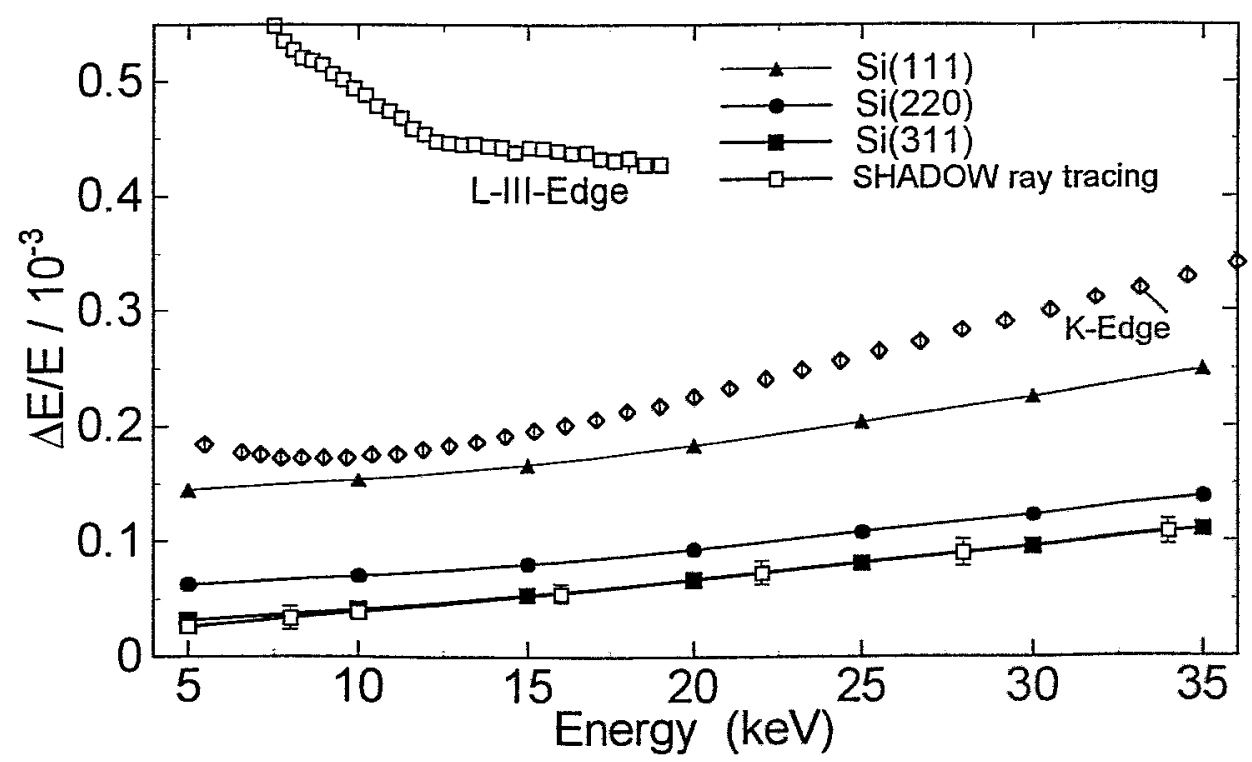

Fig. 4: Calculated energy resolution of the double crystal monochromator with silicon crystals and parallel incident beam. For comparison the natural line widths of the absorption edges of the elements are shown. Besides the analytical calculated values the result obtained with the SHADOW code is included for $\mathrm{Si}(311)$. 


\subsection{Other optical elements}

In addition to the mirrors and the monochromator, the optics contains various slit units, filters, and beam position monitors. The slit units have independently moveable blades (tungsten carbide) with an accuracy better than $10 \mu \mathrm{m}$ in horizontality and parallelism. The filter unit has six absorber foils to attenuate the white beam by different amounts. The beam position monitors consist of scanning wires.

The motions of nearly all optical components are motorised, mostly with stepper motors. They are controlled by a workstation based system using many standard ESRF software applications. For the monochromator a feedback system is installed, which compensates intensity modulations during the XAS scans. The control system includes also interlock components for the vacuum, beam shutters and the cooling of components exposed to the white beam.

Fig. 5 shows the arrangement of the beamline optics in detail. The characteristics of the monochromatic beam are summarised in Table 1.

Table 1: Characteristic data of monochromatic synchrotron beam

\begin{tabular}{|l|l|}
\hline energy range & $5-35 \mathrm{keV}$ \\
\hline \multicolumn{1}{|c|}{ energy range with Si-mirrors } & $5-12 \mathrm{keV}$ \\
\hline energy resolution with Si(111) crystals & $1.5-2.5 \times 10^{-4}$ \\
\hline energy resolution with Si(311) crystals & $0.5-1.0 \times 10^{-4}$ \\
\hline integrated flux for focussed beam (calc.) & $6 \times 10^{11} \mathrm{ph} . / \mathrm{s} \mathrm{@} 20 \mathrm{keV}, 200 \mathrm{~mA}$ \\
\hline standard beam size & $20 \mathrm{~mm}(\mathrm{w}) \times 3 \mathrm{~mm}(\mathrm{~h})$ \\
\hline focussed beam size & $\leq 0.5 \mathrm{~mm} \times 0.5 \mathrm{~mm}$ \\
\hline
\end{tabular}

\section{Radiochemistry end-station}

The radiochemistry end-station is designed for studying radionuclides which are of environmental importance such as Tc, U, Th, Np, Pu, Am and some of their decay products. A powerful technique to obtain information on the molecular and electronic structure of these radionuclides in solids and liquids is X-ray absorption spectroscopy (XAS). It is an element specific method and provides information about the oxidation state as well as the coordination and bond lengths to first, second and even third shell neighbor atoms of the absorber. Such knowledge is essential to understand complexation and speciation of radionuclides and also absorption processes from solution. Actinides are knows to exist in many oxidation states which are difficult to be determined by chemical or otical methods. In contrast XAS can clearly distinguish the oxidation state from the shift of the absorption edge and provides also information if different states are mixed in one sample. Another advantage of XAS for radiochemical investigations is the possibility to use solid or liquid samples as well as very dilute ones (micro-mol region). Such aspects 


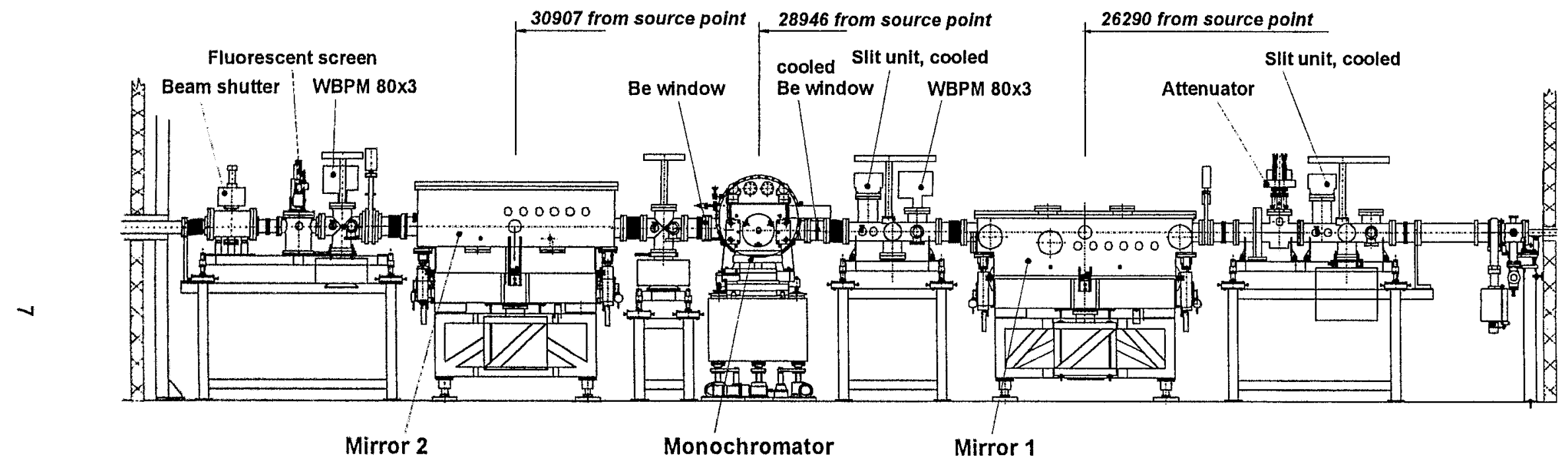

Fig. 5: Technical drawing of all components of the beamline optics. The distances of the double crystal monochromator and the mirrors from the source point are indicated. WBPM - wire beam position monitor. 
were the motivation of the FZR to set up the radiochemical part of ROBL in context of the radioecological research programme [5]

\subsection{Synchrotron radiation beamlines to study radioactive materials}

During the last five years, the number of experiments with radioactive materials performed at synchrotron radiation beamlines worldwide increased significantly. This was achieved in two ways: (i) implementation of special procedures for experiments with radioactive materials at existing general-user beamlines and (ii) construction of beamlines dedicated to the study of radioactive samples.

X-ray absorption fine structure (XAFS) spectroscopy of samples with natural uranium has been performed at several synchrotron storage rings in Europe and the US. For example at Hasylab in Hamburg, at Synchrotron Radiation Center Daresbury, at LURE, at ESRF in Grenoble, at APS in Argonne, and at SSRL in Stanford to name only a few. However, experiments with transuranics have been very limited. In 1993 a US DOE review established the fundamental basis of experimental and safety procedures for transuranic experiments at SSRL [6]. The maximum allowed quantity of ${ }^{237} \mathrm{~Np}$ in the X-ray hutch at one time is less than $50 \mu \mathrm{Ci}$.

Dedicated beamlines for the study of radioactive materials have become operational in Japan at the Photon Factory (PF) and Spring-8. The PF beamline consist of two branchlines covering the energy ranges of $1.8-6 \mathrm{keV}$ and $4-20 \mathrm{keV}$ [7]. The experimental station in the hard X-ray range is used for diffractometry, XAFS studies, and radiation biology. By a special monochromator adjustment, the energy range for XAFS experiments can be extended to $24 \mathrm{keV}$. In the soft X-ray range, the experimental possibilities to study radioactive materials have been further improved by the construction of an endstation at Spring-8 which provides linearly or circularly polarized light in the energy range $0.28-1.5 \mathrm{keV}[8]$.

The ROBL-CRG obtained a license from the French authorities to perform XAS experiments with the isotopes listed in Table 2. Under this license the maximum allowed activity at any given time present at ROBL is $185 \mathrm{MBq}(5 \mathrm{mCi})$. These elements emit mostly alpha and beta particles and only weak gamma radiation.

Table 2: List of radioactive elements which can be investigated at ROBL and the maximum amount of material to remain below the activity limit of $5 \mathrm{mCi}$

\begin{tabular}{|c|c|c|c|c|c|}
\hline Isotope & $\begin{array}{c}\text { Half-Live } \\
\text { (years) }\end{array}$ & $\begin{array}{c}\text { Amount } \\
(\mathrm{g})\end{array}$ & Isotope & $\begin{array}{c}\text { Half-Live } \\
\text { (years) }\end{array}$ & $\begin{array}{c}\text { Amount } \\
(\mathrm{g})\end{array}$ \\
\hline $\mathrm{Np} \mathrm{237}$ & $2.1 \times 10^{6}$ & 6.97 & Pu 239 & $2.4 \times 10^{4}$ & 0.08 \\
\hline Am 241 & 433 & $1.4 \times 10^{-3}$ & Pu 242 & $3.75 \times 10^{5}$ & 1.27 \\
\hline Am 243 & 7370 & 0.025 & Ra 226 & 1600 & 0.005 \\
\hline Po 208 & 2.9 & $8 \times 10^{-6}$ & Tc 99 & $2.1 \times 10^{5}$ & 29.1 \\
\hline Po 209 & 103 & $3.01 \times 10^{-4}$ & U nat & $4.47 \times 10^{9}$ & 1000 \\
\hline Pa 231 & $3.28 \times 10^{4}$ & 0.106 & Th nat & $1.4 \times 10^{10}$ & 1000 \\
\hline
\end{tabular}


Therefore, the heart of the radiochemistry end-station is a glovebox without additional lead shielding. To ensure a safe handling of the radionuclides, the entire experimental station is built as a radiochemistry laboratory according to legal safety requirements. The advantages of the ROBL radiochemistry station when compared to the other synchrotron radiation beamlines are manifold: (i) the maximum allowed amount of radioactive material is $5 \mathrm{mCi}$ which is higher than at most other synchrotron light sources, (ii) it is the only dedicated endstation for XAFS experiments of actinides in Europe. In addition, as to our knowledge, ROBL is the only XAFS station worldwide where it is possible to manipulate the chemical and physical properties of the sample in a glove box on site and during the experiment at a third-generation high-energy synchrotron radiation source.

\subsection{Experimental set-up for XAS}

The XAS spectrum of the samples can be measured both in transmission and fluorescence modes. For transmission mode the sample is placed perpendicular to the photon beam between two ionisation chambers (OHOYO KOKEN KOGYO). If the XAS spectrum is measured in fluorescence mode, the sample is inclined by $45^{\circ}$ with respect to the beam to collect the fluorescence radiation with a $\mathrm{Ge}$ solid state detector (Lawrence Berkeley National Laboratory) positioned perpendicular to the beam. The radioactive samples are positioned inside the glovebox equipped with $125 \mu \mathrm{m}$ thick Kapton polyimide windows which are transparent to hard X-rays. All detectors, e.g., gas ionisation chambers and fluorescence detectors, are mounted on an optical bench outside the glovebox. This arrangement allows a direct and easy access to the detectors and avoids bringing signal cables and gas and power supply lines into the glovebox. In addition, even in the unlikely case of a contamination inside the glove box, the detectors outside the glovebox will not be affected.

Inside the glovebox it is possible to use different sample positioners depending on the type of sample. Two sample positions marked (a) and (b) are indicated in the schematic glovebox layout in Fig. 6 . At the larger position (a) it is possible to mount either an automatic positioner holding up to eight solid or liquid samples or a positioner for the closed cycle He cryostat. The samples in position (a) can be measured both in transmission and fluorescence modes depending on the radionuclide concentration. For the measurement of very dilute samples, a special sample holder for a single sample can be placed at position (a). This positioner has a rotating arm and moves the sample to position (b). In this position the sample is placed between two Kapton windows and inclined $45^{\circ}$ with respect to the beam allowing two Ge solid state detectors to record simultaneously the fluorescence radiation from the sample. Most of the motion axes of the described sample positioners are motorised and remote controlled.

Since the samples are safely contained in the glove box, it is possible to change some of the sample conditions during the experiment. With the closed cycle He cryostat (custom designed from Oxford Instruments) the sample 


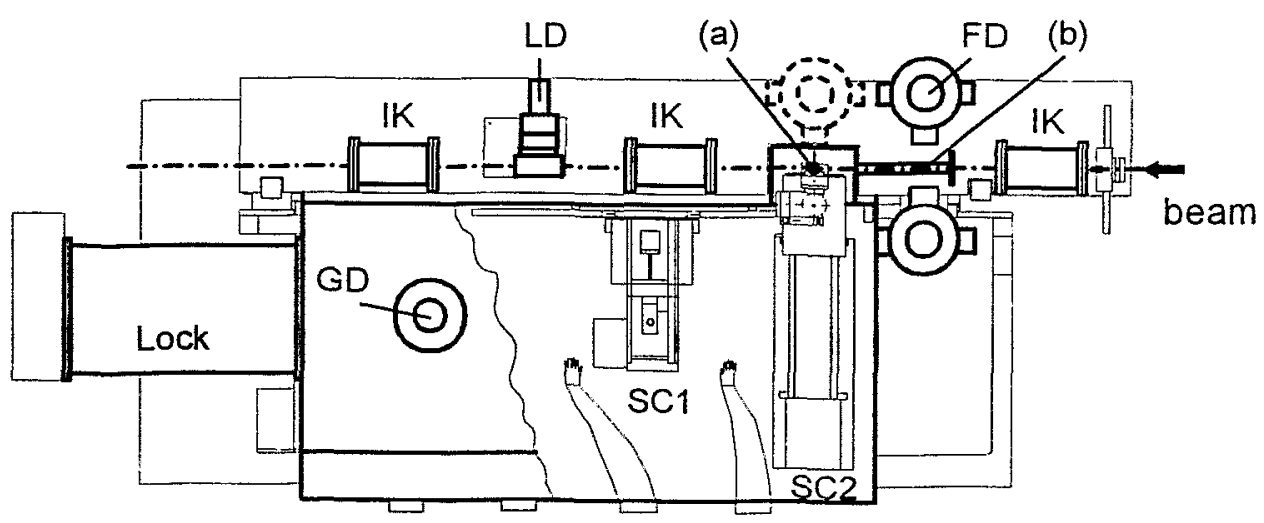

Fig. 6: Principal layout of the glovebox. (a) standard sample position for XAS experiments; (b) sample position for fluorescence radiation detection from dilute liquid samples. The ionisation chambers (IK) are mounted on an optical bench and can also be used for non-radioactive samples while the box is moved out of the beam. FD-fluorescence detector; LD - Lytle detector; GD - gamma detector for activity control.

temperature can be varied between 10 and $295 \mathrm{~K}$. It is also possible to modify the chemical conditions of liquid samples just before or during the XAS measurements by adding non-radioactive substances like acid, base or complexing agents.

As mentioned before all the detectors are mounted outside the glovebox on an optical bench. For energy calibration purpose a non-radioactive reference sample can be placed between the second and third gas ionisation chamber. The reference sample is inside a fluorescence X-ray ion chamber detector (Lytle detector; EXAFS Company) to record the fluorescence XAS if measurement in transmission is not possible.

The glovebox is mounted on a support frame which allows to move the glovebox in horizontal direction out of the beam leaving the position of the optical bench and the detectors unchanged. This has the advantage that nonradioactive samples can be easily measured outside the glovebox by mounting them on the optical bench between the first and second gas ionisation chamber.

When measurements take place downstream in $\mathrm{MRH}$, the beam path in $\mathrm{RCH}$ is closed by a vacuum pipe to reduce the loss of beam intensity. For this purpose the box will be moved horizontally and the optical bench vertically out of the beam. 


\subsection{Safety concept for radioactive work}

In order to guarantee a safe operation of the experiments with radioactive samples a number of safety installations was made to monitor the actual status. Basically a multi-barrier concept is realised here as usual for radiochemical work. The scheme of this concept is given in Fig. 7. The samples are enclosed in a X-ray transparent container serving as the first barrier. The sample container itself is either inside a multi-walled transport container or within the glovebox. The glovebox is the second barrier under experiment conditions. The glovebox is located in the hutch which is a third barrier in regard to the experimental hall of the ESRF. For this, the radiochemistry hutch is equipped with a closed inner wall. It is made from steel panels and the connections between the panels are sealed by epoxy resin. The two functions to be achieved are: (i) a good air tightness of the hutch (to allow for a pressure gradient to the hall) and (ii) to provide a smooth surface (for decontamination). The condition of air tightness of the $\mathrm{RCH}$ demands to have the incoming synchrotron radiation beam always in the same position. This is realised by the optics layout as discussed in paragraph 2 . The floor has a highly resistant plastic covering. The equipment is mounted on plates. The interface between the floor covering and the walls as well as the mounting plates are sealed by epoxy resin.

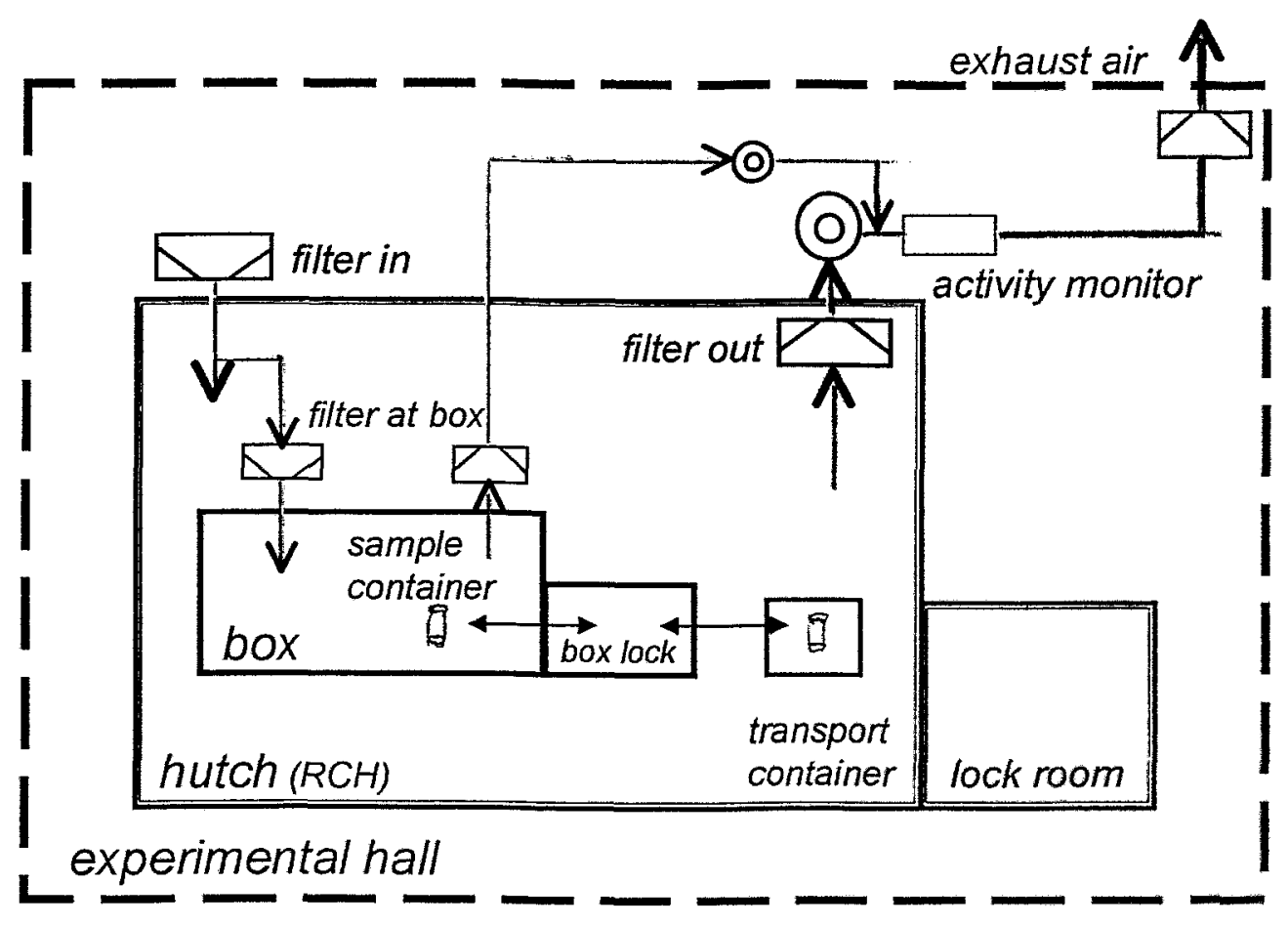

Fig. 7: Scheme of the multi-barrier concept for the safety system of the radiochemistry hutch of $R O B L$. 
The negative pressure gradient from hall to hutch and from hutch to glovebox will be established by a special air ventilation system for the $\mathrm{RCH}$. This system is independent of all other systems and performs 8 complete volume changes of air per hour. Separate ventilators for the glove box and the hutch exhaust air, each with high absorption absolute aerosol filters, ensure for an automatically regulated pressure difference of $200 \mathrm{~Pa}$ between glovebox and hutch. The exhaust air will be controlled by a permanent monitoring system outside the $\mathrm{RCH}$ to be free from radiation background effects. Before the filtered and monitored exhaust air leaves the experimental hall over the roof, a final filter is inserted serving as a last barrier to the environment.

The personnel access to $\mathrm{RCH}$ is possible only through the lock room (RCL in Fig. 1). There a change of coats and shoes has to be performed. After leaving the $\mathrm{RCH}$ the experimenters have to check themselves with the personnel monitor for possible contamination and to change clothing again.

The $\alpha / \beta$-activity as well as the dose rate of the air inside the $\mathrm{RCH}$ is permanently controlled by a moving filter aerosol monitor. All safety related data are continuously collected in a signalisation system, which optically and acoustically indicates all changes, failures and deviations from reference values within the safety system. In case of an emergency in the $\mathrm{RCH}$ the signalisation system will cause to close the beam shutter and the vacuum valves via the ESRF interlock system.

For the identification and documentation of individual samples a $\gamma$-spectrometer is mounted on the floor inside the glovebox (GD in Fig.6). A hand-foot monitor in the lock room, movable $\alpha / \beta$ and dose rate counters and a fixed alpha/beta measuring station complete the safety equipment of the $\mathrm{RCH}$.

The preparation of radioactive samples is not possible at the ESRF. Samples will be transported to the experimental end-station in certified "transport containers" made from steel. It contains a "working container" equipped with 1 $\mathrm{cm}$ lead walls absorbing all radioactive radiation up to a limit of $5 \mu \mathrm{Sv} / \mathrm{h}$. This working container will be opened only in the glovebox and the polyethylene "sample container" can be removed.

\subsection{Performance tests by XAFS spectroscopy}

To check the energy calibration of the Si(111) double crystal monochromator, the X-ray absorption near-edge structure (XANES) spectra of several metal foils in the energy range of $5-30 \mathrm{keV}$ was measured in transmission mode. The obtained energies of absorption edges of $\mathrm{Ti}, \mathrm{Cr}, \mathrm{Co}, \mathrm{Zn}, \mathrm{Zr}, \mathrm{Nb}$, and $\mathrm{Sb}$ foils were compared with the values given in [9]. The data evaluation showed that the standard deviation of the Bragg angle was less than $0.002^{\circ}$ in the entire energy range indicating an excellent linearity of the monochromator mechanics. Figure 8 displays single sweeps of K-edge XANES spectra of three representative elements. The features of the $\mathrm{Ti}$ and $\mathrm{Zr}$ K-edges are well resolved. From the experiments one can conclude that the ability to resolve 
individual transitions in the XANES region is limited by the natural core-hole lifetime and not by the resolution of our Si(111) DCM.

The performance of the whole beamline optics and detector system is also demonstrated by the high quality of the Tc K-edge $k^{3}$-weighted extended $\mathrm{x}$-ray absorption fine structure (EXAFS) spectrum given in Fig. 9. This spectrum of a $0.1 \mathrm{Mol} / \mathrm{L} \mathrm{Tc}(\mathrm{VII})$ solution is a single sweep recorded in transmission mode. At the beginning of the EXAFS region the counting time per point was one second. The measuring time was continuously increased with $k$ and reached 20 sec per point at $k$ of $20 \AA^{-1}$.

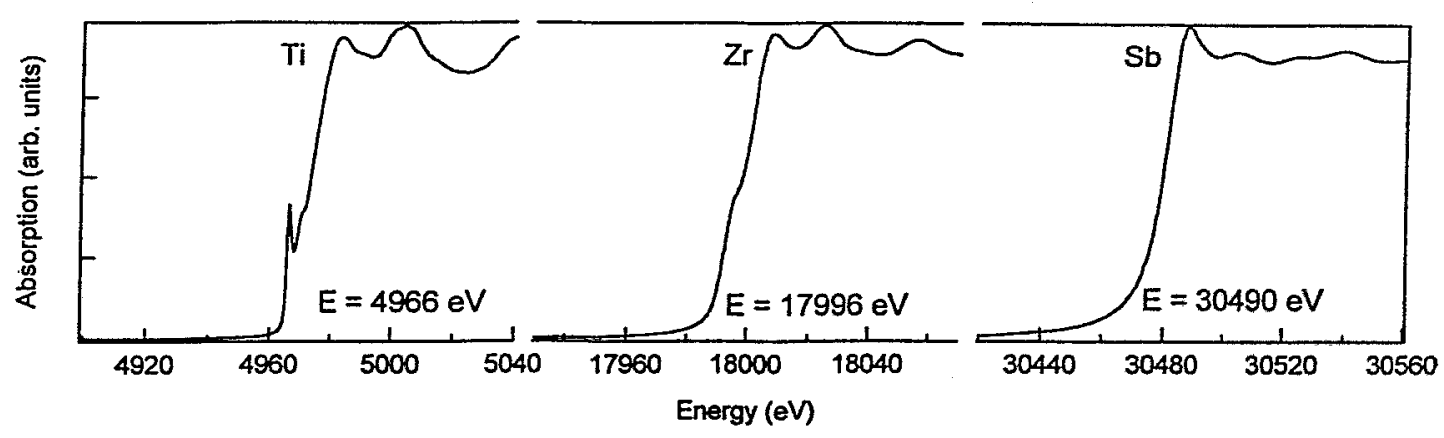

Fig. 8: K-edge XANES spectra of metallic $\mathrm{Ti}, \mathrm{Zr}$, and $\mathrm{Sb}$ (from left to right). The edge energies are from [9]. The natural line widths of the $\mathrm{Ti}, \mathrm{Zr}$, and $\mathrm{Sb} \mathrm{K}$ shells are $0.94,3.84$, and $9.16 \mathrm{eV}$, respectively [10].

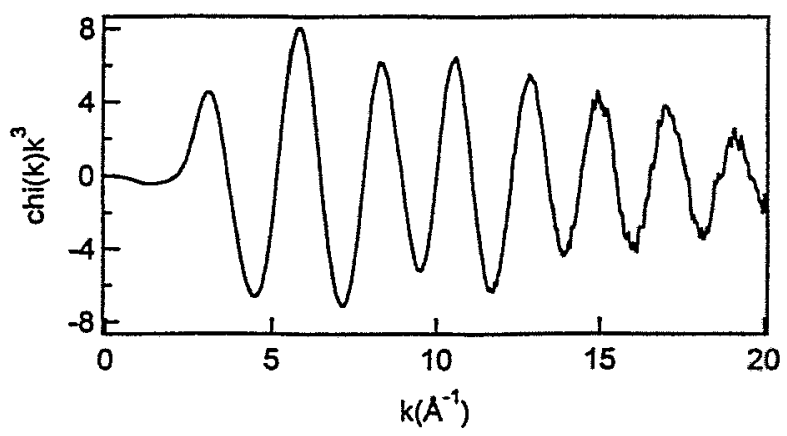

Fig. 9: Single sweep of the Tc K-edge $k^{3}$-weighted EXAFS spectrum of a 0.1 $\mathrm{Mol} / \mathrm{L}$ pertechnetate, $\mathrm{TCO}_{4}{ }^{\circ}$, solution. 


\section{Materials research end-station}

The materials research end-station is mainly intended for structural studies of solids and melts by diffraction and reflectometry. Of special interest are structures in thin near-surface regions of solids as this is the typical modification region for samples treated by ion beam techniques. The diffractometer is versatile enough to allow for a wider range of applications of this end-station. A special design was made for the study of melts with free surfaces by diffraction techniques.

\subsection{Goniometer}

The goniometer (Fig. 10) as the main instrument in the MRH is a six-circle design built from standard components (Huber). The arrangement of two parallel circles each with horizontal and vertical axes, respectively, allows experiments in both scattering planes. The sample position can be equipped with an $x-y-z$-slide or, alternatively, with special sample environment chambers which are mounted directly to the $\phi$-circle. The layout is made for a load up to $15 \mathrm{~kg}$ at the sample position and the $\chi$-circle has an inner diameter of $370 \mathrm{~mm}$ so that even huge and heavy chambers (e.g. a high temperature chamber) can be operated without difficulties. All axes are equipped with stepper motors and gear boxes which allow a minimal angular step of $0.0001^{\circ}$. The z-translation of the $x-y-z$-slide has a step width of $1 \mu \mathrm{m}$ while the two other translations have 10 $\mu \mathrm{m}$. Additionally, sample holders for single crystal samples and capillary sample containers are available.

The goniometer is fully computer controlled by a workstation and the programs are based on the SPEC ${ }^{\hat{G}}$ code.

Different detection systems can be mounted at the detector arm. As standard, a high load high linearity scintillation detector is used (BEDE). In front of the detector, interchangeable fixed single or soller slits can be mounted. Other detection systems are a two dimensional CCD-camera (SMART) or an energydispersive pin-photodiode. Optionally a secondary monochromator unit in front of the detector may be mounted on the detector arm.

\subsection{Resolution for $X$-ray diffraction}

In contrast to XAS experiments the resolution of a diffraction experiment is not only determined by the properties of the monochromator but depends also on the beam divergence. The experimentally achievable resolution was determined with silicon single crystals and powders. At present, radiation with the natural horizontal divergence of the synchrotron radiation beam from the source is used since the sagittally focussing option at the monochromator is not yet installed. The vertical divergence is reduced by the first collimating mirror.

Using the Si(111) monochromator and the Si stripes of the mirrors in the optics, at $12 \mathrm{keV}$ and with a vertically parallel beam the FWHM of a Si(004) single 


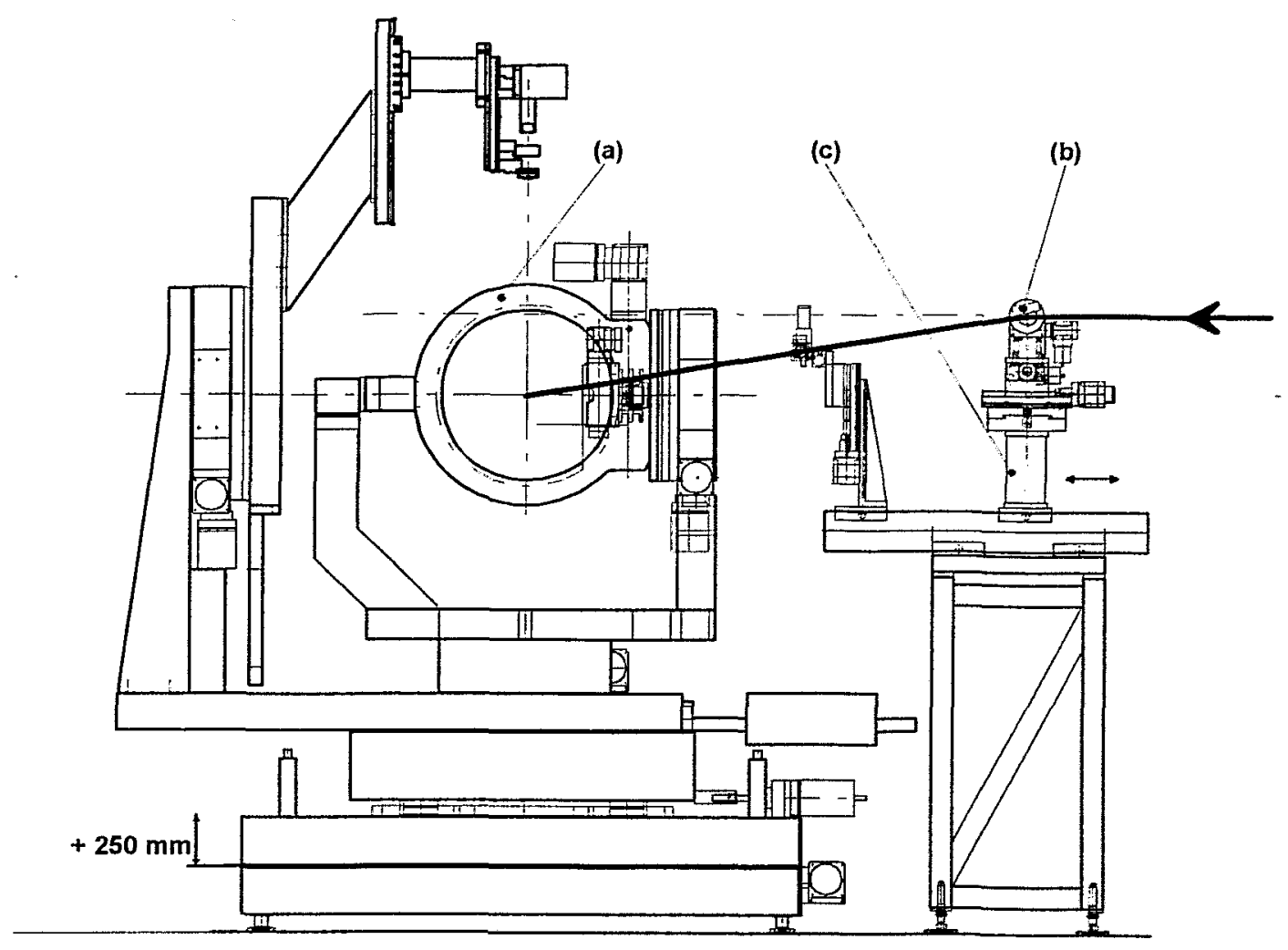

Fig. 10: Scheme of the goniometer (a) in the materials research end-station and arrangement of deflector unit for the study of melts with free surfaces. The small goniometer head with the deflecting multilayer mirror (b) can be positioned in different distances to the sample by a translation slide (c). The whole sample goniometer can be lifted up and down (stroke 250 $\mathrm{mm}$ ) to bring the sample into the appropriate height to hit the beam.

crystal reflection was measured to be $\Delta \vartheta=0.003^{\circ}$ (10 arcsec). This corresponds approximately to a resolution $\Delta \mathrm{d} / \mathrm{d}=1.3 \times 10^{-4}$. Vertical focussing with the second mirror raises the FWHM up to $0.0051^{\circ}$ but increases the incident radiation intensity significantly. Fig. 11 shows the FWHM of a Si(004) reflection and the monitor count rate in dependence on the inverse radius of curvator of mirror M2. It follows from these results, that ROBL's optics allows optimizing the intensity by vertical focussing without significant loss in resolution.

With respect to powder diffraction, in comparison to the contribution from powder samples the instrumental contribution to the intrinsic FWHM of Bragg peaks is negligible, as was stated also for the ESRF powder diffraction beamline BM16 [11]. A comparison of powder with single crystal results for the $\mathrm{Si}(004)$ reflection at an incident energy of $14 \mathrm{keV}$ in Fig. 12 shows that essentially the crystallite properties of the powder determine the experimental FWHM-values. The higher FWHM-values for the single crystal reflection are due to the higher energy of radiation, to the use of Pt/Pt-mirrors, and to the fact 

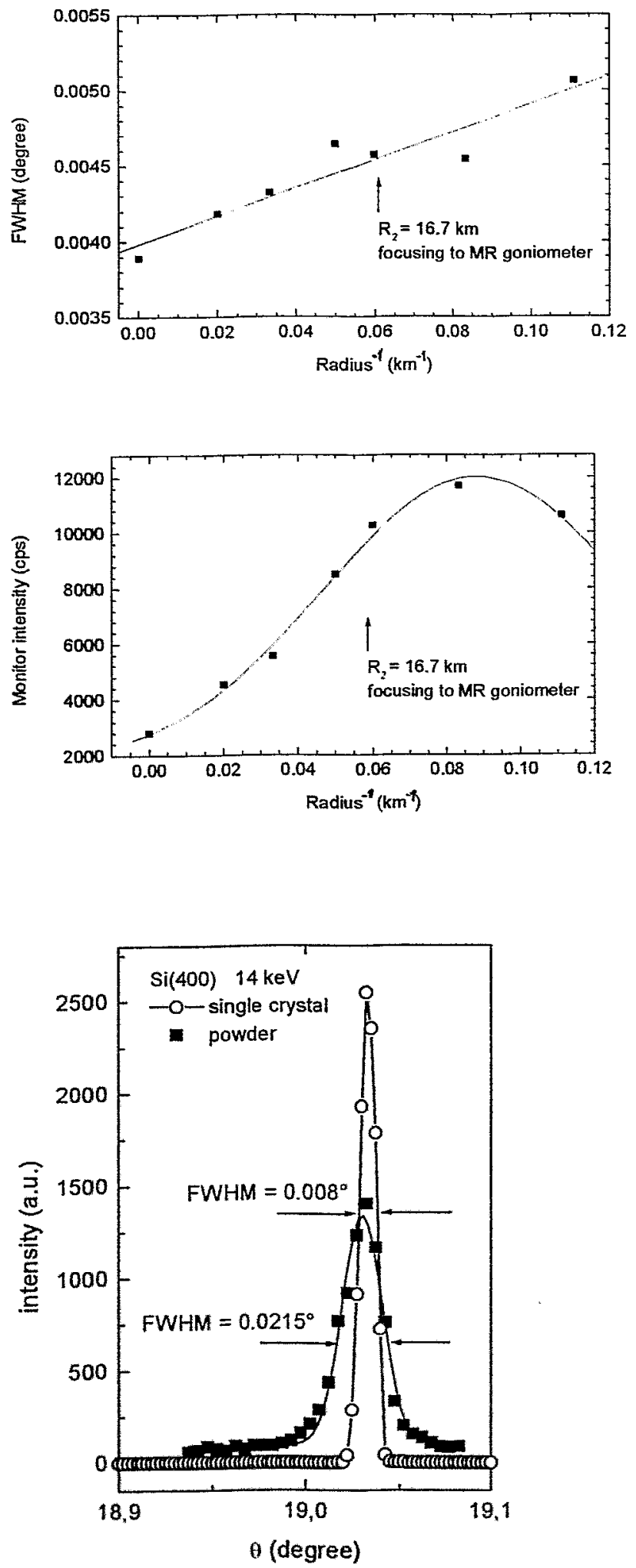

Fig. 11

a) Divergence of the beam to the MRH goniometer as measured by the rocking curve FWHM of the Si(400) single crystal reflection in dependence on the bending of mirror $\mathrm{M} 2$ (Bending radius of mirror M1 $=20.93 \mathrm{~km}$, wavelength $\lambda=0.10008 \mathrm{~nm}$ )

The given result is the FWHM of the substrate peak of $\mathrm{Si}<100>$ implanted with $5^{*} 10^{15} \mathrm{C}^{+}$ $(195 \mathrm{keV}) \mathrm{cm}^{-2}$ at $500{ }^{\circ} \mathrm{C}$.

b) Intensity of the incident beam to the MRH goniometer (measured as monitor intensity) in dependence on the bending of mirror $\mathrm{M} 2$ at same conditions.

Fig. 12

Comparison of the FWHM of the $\mathrm{Si}(400)$ reflection measured as rocking curve from a single crystal and from powder diffraction. 
that the sample crystal is non-ideal. Typical Si-powder resolution curves $\Delta \mathrm{d} / \mathrm{d}$ for different energies and monochromator crystals are given in Fig. 13. These data show that the materials research end-station is also suitable for high resolution X-ray diffraction and for the study of line broadening effects [12].

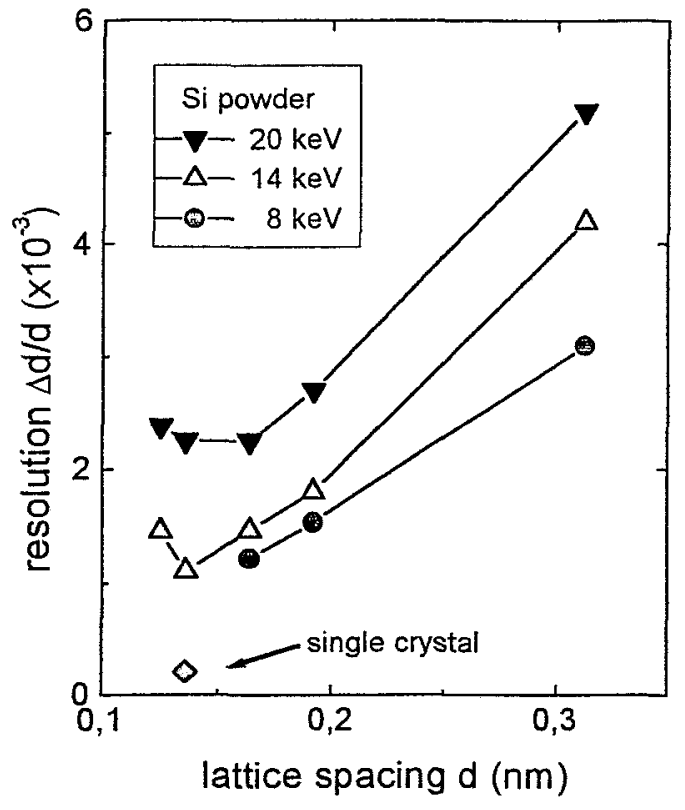

Fig. 13

Experimentally determined diffraction resolution $\Delta \mathrm{d} / \mathrm{d}$ of a silicon powder inside a glass capillary. For comparison the value from a single crystal sample is indicated.

\subsection{Special set-up for the study of melts}

One of the design goals was to have the opportunity for structural studies of melts (or more general liquids) with free surfaces. This requires an incident synchrotron radiation beam inclined to the horizontal in order to realize an angle of incidence above the critical angle of total reflection on the horizontal surface of the melt. The additional demand to tune the energy in order to make use of anomalous scattering led to the set-up displayed in Fig. 10. In front of the sample at the goniometer (a) a deflection unit (b) is installed. A suitable multilayer of $40 \times 100 \mathrm{~mm}^{2}$ is mounted on a circle with a horizontal rotation axis perpendicular to the incident beam. This deflects the beam downwards. The deflecting multilayer mirror made from $W / B_{4} C$ with a $2 d$-value of $3 \mathrm{~nm}$ (Osmic). The measured reflecting efficiency of the mirror is $50 \%$ at $8 \mathrm{keV}$. In order to hit the sample with the deflected beam the goniometer has a motorised height adjustment with a stroke of $250 \mathrm{~mm}$ below the primary beam from the monochromator.

In case of change of the energy of the incident beam the deflection angle of the mirror and the height of the sample have to be adjusted. In order to reduce the height adjustment cycles of the huge diffractometer, an additional translation stage (c) is situated below the multilayer-mirror. With a fixed sample height the 
adjustment is achievable by translation and rotation of the deflecting mirror alone for a energy variation of $5 \mathrm{keV}$ in the low-energy range centered at 10 $\mathrm{keV}$ and up to $11 \mathrm{keV}$ in the high-energy range centered at $25 \mathrm{keV}$.

\subsection{Comparison to other beamlines}

The material research hutch (ROBL-MRH) opens additional possibilities in Xray diffraction on polycrystals and liquids at the ESRF. The dedicated ESRF powder diffraction beamline (BM16) as well as the powder diffraction setup of Swiss-Norwegian-Beamline (BM1) are equipped with goniometers which are restricted to one scattering plane $[11,13,14]$. ROBL-MRH offers horizontal and vertical scattering planes as well as off-plane diffraction possibilities. For powder or polycrystalline samples we provide the same diffraction resolution and nearly the same wavelength range as the mentioned beamlines. Other goniometers at the CRG Beamlines "Interfaces" (BM32) [15] and "DUBBLE" (BM26) [16] are specialized designs for the study of epitaxial films. Therefore they have an additional seventh axis for sample orientation [e.g. 17]. Since ROBL-MRH is mainly devoted to structural studies of polycrystalline thin films such additional axis is not necessary. Concerning the study of liquids with free surfaces the ROBL-MRH setup described in 4.3. is complementary to the BM32. The latter uses a silicon crystal in Laue geometry as beam deflector [18]. This arrangement requires the movement of the goniometer in two directions to follow the Bragg cone of the radiation scattered from the deflector. The use of a multilayer as deflector at ROBL-MRH limits the movements of the goniometer to the up-down-direction which is a more simple design. It provides the same possibilities but makes the arrangement of samples in chambers with small radiation windows easier.

\section{Outlook}

The ROBL beamline with both end-stations become fully operational in fall 1998. First experiments show a reliable performance of the whole complex. This CRG beamline adds new experimental possibilities to the ESRF. The radiochemistry end-station is the first dedicated set-up for XAS studies of unsealed liquid radioactive samples at a modern synchrotron source. The materials research end-station allows diffraction in both scattering planes at a goniometer which can carry heavy sample chambers.

\section{Acknowledgements}

The authors acknowledge the support by the management of the FZR which makes the project possible. ROBL is financed by equal shares from the Saxonian Ministry of Science and Culture and the Federal Ministry of Research and Education via the budget of the FZR. 
Great support was given by colleagues from the ESRF and the other CRG beamlines. We want to thank in particular: I. Kilvington, $P$. Pattison, $P$. Berkvens, M. Belakhovsky, A. Fitch, E. Bräuer-Krisch, M. Hagelstein, D. Schmied, G. Peppelin.

\section{References}

[1] ESRF Beamline handbook, Grenoble, August 1993, p. 9-10

[2] T. Mairs, ESRF machine division, private communication

[3] D.Pauschinger, K.Becker, and R.Ludewig, Rev.Sci.Instr. 66 (1995) 2177

[4] W. Matz, F. Prokert, R. Schlenk, J. Claußner, N. Schell, F. Eichhorn, and G. Bernhard, The Rossendorf Beamline at the ESRF (Project ROBL), internal report FZR-158, Rossendorf 1996

B. Lai, F. Cerrina, Nucl. Instr. \& Methods, A246 (1986) 337

[5] H. Nitsche, T. Reich, C. Hennig, A. Roßberg, G. Geipel, M.A. Denecke, L.Baraniak, P. Panak, A. Abraham, B. Mack, S. Selensk-Pobell, G. Bernhard, Proc. Euroconference/NEA-Workshop Actinide-XAS-98, October 4-6, 1998, Grenoble, France, NEA, Paris 1999, in press

[6] P.G. Allen, D.K. Shuh, J.J. Bucher, N.M. Edelstein, Proc. Euroconference/NEA-Workshop Actinide-XAS-98, October 4-6, 1998, Grenoble, France, NEA, Paris 1999, in press

[7] H. Konishi, A. Yokoya, H. Shiwaku, H. Motohashi, T. Makita, Y, Kashihara, S. Hashimoto, T. Harami, T.A. Sasaki, H. Maeta, H. Ohno, H. Maezawa, S. Asaoka, H. Kanaya, K. Ito, N. Usami \& K. Kobayashi, Nucl. Instrum. Methods A 372 (1996) 322-332

[8] A. Yokoya, T. Sekiguchi, Y. Saitoh, T. Okane, T. Nakatani, T. Shimada, H. Kobayashi, M. Takao, Y. Teraoka, Y. Hayashi, S. Sasaki, Y. Miyahara, T. Harami \& T.A. Sasaki, J. Synchrotron Rad. 5 (1998) 10-16

[9] S. Kraft, J. Stümpel, P. Becker, and U. Kuetgens, Rev. Sci. Instrum. 67 (1996) $681-687$

[10] M. O. Krause and J. H. Oliver, J. Phys. Chem. Ref. Data 8 (1979) 329

[11] A.N. Fitch, Materials Science Forum 228-231 (1996) 219-222

[12] E. Thiele, M. Hecker, N. Schell, Proc. $6^{\text {th }}$ EPDIC, Budapest, August 
$22-25,1998$, to be published in Materials Science Forum

[13] A.N. Fitch, Nucl. Instr. and Meth. in Phys. Res. B 97 (1995) 63-69

[14] ESRF Beamline handbook, fourth ed., Grenoble, January 1997, p. 25-29

[15] ESRF Beamline handbook, fourth ed., Grenoble, January 1997, p. 201206

[16] M. Borsboom, W. Bras, I. Cerjak, D. Detollenaere, D. Glastra van Loon, P. Goedtkindt, M. Konijnenburg, P. Lassing, Y. K. Levine, B. Munneke, M. Oversluizen, R. van Tol, E. Vlieg, J. Synchr. Rad. 5 (1998) 518-520 W. Bras, J. Macromol. Sci.-Phys., B37 (1998) 557-565

[17] E. Vlieg, J. Appl. Cryst. 31 (1998) 198-203

[18] J. Daillant, K. Quinn, C. Gourier, F. Rieutord, J. Chem. Soc., Faraday Trans., 92 (1996) 505-513 